\title{
Complex Binary Adder Designs and their Hardware Implementations
}

\author{
Tariq Jamil ${ }^{1}$, Medhat Awadalla ${ }^{2}$, Iftaquaruddin Mohammed $^{3}$ \\ Department of Electrical and Computer Engineering \\ Sultan Qaboos University \\ AlKhod, OMAN
}

\begin{abstract}
Complex Binary Number System (CBNS) is $(-1+\mathbf{j})$ based on binary number system which facilitates both real and imaginary components of a complex number to be represented as single binary number. In this paper, we have presented three designs of nibble-size complex binary adders (ripple-carry, decoder-based, minimum-delay) and implemented them on various Xilinx FPGAs. The designs of base2 4-bit binary adder have also been implemented so that statistics of different adders can be compared.
\end{abstract}

Keywords-Complex number; complex binary; adder; ripple carry; decoder; minimum delay

\section{INTRODUCTION}

Complex numbers play important roles in various areas of electrical and computer engineering but their representation and treatment in the realm of computing are based on a divideand-conquer technique wherein real part of the complex number is dealt with separately and imaginary part of the complex number is handled separately. Thus, addition of two complex numbers $(\mathrm{a}+\mathrm{jb})$ and $(\mathrm{c}+\mathrm{jd})$ involves two separate additions: $(a+c)$ for the real parts and $(b+d)$ for the imaginary parts. To facilitate single-unit representation of a complex number which will ultimately result in the reduction of arithmetic operations for the real and imaginary components of complex numbers, Complex Binary Number System (CBNS) with $(-1+j)$-base has been proposed in the scientific literature [1-4]. In this paper, we are going to present three designs of nibble-size complex binary adder circuits and their implementations on various Xilinx FPGAs. For the sake of comparison, we'll also present implementation of base 2 nibblesize adder so that relative complexity of different adder designs can be appreciated.

\section{COMPLEX BinARY NumBer System}

\section{A. Binary Representation}

The value of an n-bit binary number with base $(-1+\mathrm{j})$ can be written in the form of a power series as follows: $a_{n-1}(-1+j)^{n-1}+$ $a_{n-2}(-1+j)^{n-2}+a_{n-3}(-1+j)^{n-3}+\ldots+a_{2}(-1+j)^{2}+a_{1}(-1+j)^{1}+a_{0}(-1+j)^{0}$ where the coefficients $a_{n-1}, a_{n-2}, a_{n-3}, \ldots, a_{2}, a_{1}, a_{0}$ are binary (either 0 or 1 ). This is analogous to the ordinary binary number system power series of $a_{n-1}(2)^{n-1}+a_{n-2}(2)^{n-2}+a_{n-3}(2)^{n-3}+\ldots+a_{2}(2)^{2}$ $+a_{1}(2)^{1}+a_{0}(2)^{0}$ except that the bases are different. Details about how to convert a given complex number into $(-1+\mathrm{j})$-base complex binary number representation can be found in [1-4]. By the application of the conversion algorithms mentioned in these publications, a given complex number can be represented as a single binary entity. For example, the complex number $2019+j 2019$ has the binary representation in base $(-1+j)$ as: 1110100000001110100001100110.

\section{B. Addition Algorithm}

The binary addition of two complex binary numbers follows these rules: $0+0=0 ; 0+1=1 ; 1+0=1 ; 1+1=$ 1100. These rules are very similar to the traditional binary arithmetic except for the last case when two numbers with $1 \mathrm{~s}$ in position $n$ are added, this will result in $1 \mathrm{~s}$ in positions $n+3$ and $n+2$ and 0 s in positions $n+1$ and $n$ in the sum. Similar to the ordinary computer rule where $1+111 \ldots$ (to limit of machine) $=0$, we have $11+111=0$ [Zero Rule $]$.

\section{ADDER DESIGNS}

\section{A. Ripple-Carry}

The block diagram of a 4-bit Complex Binary Ripple-Carry Adder (CBRCA) is shown in Fig. 1 [5].

The adder performs the addition of two 4-bit complex binary numbers $\mathrm{A}\left(a_{3} a_{2} a_{1} a_{0}\right)$ and $\mathrm{B}\left(b_{3} b_{2} b_{1} b_{0}\right)$ and generates a 4-bit $(-1+\mathrm{j})$-radix result (Sum) and up to 8 Extended-Carries. It consists of the Addition Unit, the Extended-Carry Generation Unit, the Zero Detection Unit, and the Output Generation Unit.

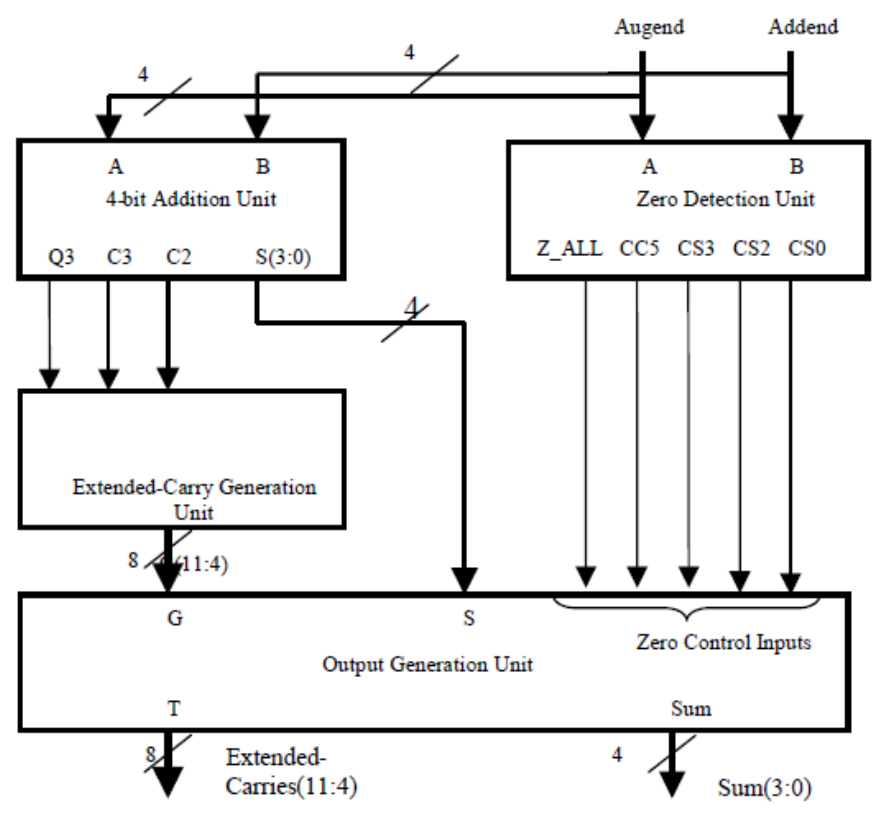

Fig. 1. Block Diagram of a 4-bit Complex Binary Ripple-Carry Adder [5]. 
The Addition Unit is structured from 4 semi-cascaded stages. Each stage is responsible of generating one of the bits of the result $\left(S_{0}-S_{3}\right)$. The carry generated from the addition of two bits in $(-1+\mathrm{j})$ radix representation at stage $i$ produces a carry that should be propagated to stages $i+2$ and $i+3$. Since no carry-in(s) to the adder is assumed, stages 0 and 1 are easily implemented using two half-adders. Stage 2 is implemented using a full-adder with a carry-in generated from stage 0 . While for stage 3, a specially designed 4-input binary variables adding component is implemented [6]. Stage 3 performs the addition of bits $b_{3}$ and $a_{3}$ of the Addend and Augend with possible two carries referred to by $K_{31}$ and $K_{32}$, which may be generated from stages 0 and 1 , respectively. Stage 3 produces bit $S_{3}$ of the result and two carry bits, $C_{3}$ and $Q_{3}$, according to the 4 binary variables truth table for the addition stage. $C_{3}$ is a normal carry due to adding three ones $(1+1+1)$, and $Q_{3}$ is an extended carry due to adding four ones $(1+1+1+1)$ in $(-1+j)$ radix representation. $C_{3}$ should propagate to stages 5 and 6 , and $Q_{3}$ to stages 7, 9, 10, and 11. Since the adder performs 4-bit ($1+\mathrm{j}$ )-base complex number addition, the carries $C_{2}, C_{3}$, and $Q_{3}$ are taken to the inputs of the Extended-Carries unit, in order to generate all the necessary carries. All carries generated by stages 2 and 3 are handled by dummy stages in the ExtendedCarry Generation Unit, referred to by stages 4 to 11 . Each stage of the Extended-Carries Unit is responsible of generating one Extended-Carry bit. The Boolean equations for stages 0,1 , and 2 are obvious from the use of half-adders and full-adder circuits. For stage 3, the Boolean equations of the outputs are found from the minimization of 4-variable Karnaugh maps. These are,

$$
\begin{aligned}
S_{3}= & a_{3} \oplus b_{3} \oplus K_{31} \oplus K_{32} \\
C_{3}= & \bar{a}_{3} K_{31} K_{32}+b_{3} K_{31} \bar{K}_{32}+a_{3} \bar{b}_{3} K_{31}+ \\
& a_{3} \bar{K}_{31} K_{32}+a_{3} b_{3} \bar{K}_{31}+b_{3} \bar{K}_{31} K_{32} \\
Q_{3}= & a_{3} b_{3} K_{31} K_{32}
\end{aligned}
$$

The $S_{3}$ expression is a 4-input odd function that can be implemented by EXCLUSIVE-OR gates, the $Q_{3}$ expression is a 4-input AND function, and the $C_{3}$ is a sum-of-product expression that can be implemented by a two-stage logic (e.g., AND-OR, or NAND-NAND).

The Extended-Carry Generation Unit consists of 8 dummy stages, 4-11. They handle the propagated carries from stages 2 $\left(C_{2}\right)$ and $3\left(C_{3}, Q_{3}\right)$ in the Addition Unit. The Dummy stages 4, 5,6 , and 8 are implemented using half adders, and dummy stages 7, 9, 10, and 11 are implemented using full-adders. The unit would generate the extended carries $\left(C_{4}-C_{11}\right)$ as inputs to the Output Generation Unit.

The Zero Detection Unit determines the conditions necessary to generate special output results based on the recognition of specific patterns for the Addend and the Augend. All conditions considered are based on the Zero Rule for the $(-1+\mathrm{j})$ radix number representation. Assuming 4-bit ($1+\mathrm{j})$ radix numbers, the unit receives inputs for the Addend $\left(b_{3}\right.$ $\left.\begin{array}{lll}b_{2} & b_{1} & b_{0}\end{array}\right)$ and the Augend $\left(\begin{array}{llll}a_{3} & a_{2} & a_{1} & a_{0}\end{array}\right)$, and generates five control signals: $C S O, C S 1, C S 3, C C 5$, and $Z \_A L L$. The five control signals are generated based on the patterns detected for the Addend $\left(b_{3} b_{2} b_{1} b_{0}\right)$ and the Augend $\left(a_{3} a_{2} a_{1} a_{0}\right)$, which satisfy the Zero Rule. Table 1 lists all the minterms of the input that will generate special output results.

The Boolean expressions characterizing each control output are defined below.

1) CSO Control Output: CSO controls the summation bit $S_{0}$ according to table 1 . Its Boolean expression is described as:

$$
\begin{gathered}
C S 0=\sum(111,126,231,239,246,254) \\
C S 0=\left(a_{2} a_{1} b_{2} b_{1}\right)\left(\overline{a_{0}} b_{3} b_{0}+a_{0} b_{3} \overline{b_{0}}+\right. \\
\left.a_{3} b_{3}\left(a_{0} \oplus b_{0}\right)\right)
\end{gathered}
$$

2) CS2 Control Output: $C S 2$ controls the summation bit $S_{2}$ according to table 1. Its Boolean expression is described as:

$$
\begin{aligned}
& C S 2=\sum(119,127,247,255) \\
& C S 2=\left(a_{2} a_{1} a_{0} b_{2} b_{1} b_{0}\right)
\end{aligned}
$$

3) CS3 Control Output: $C S 3$ controls the summation bit $S_{3}$ according to table 1. Its Boolean expression is described as:

$$
\begin{aligned}
C S 3= & \sum \begin{array}{l}
(63,123,127,183,238,239 \\
243,247,254)
\end{array} \\
C S 3= & \left(a_{1} b_{1}\right)\left(\overline{a_{3}} a_{0} b_{3} b_{2} b_{0}+a_{3} a_{0} \overline{b_{3}} b_{2} b_{0}+\right. \\
& a_{3} a_{2} b_{3} b_{2} \overline{b_{0}}+\overline{a_{3}} a_{2} a_{0} b_{3} b_{0}+ \\
& \left.a_{3} a_{2} a_{0} \overline{b_{3}} b_{0}+a_{3} a_{2} \overline{a_{0}} b_{3} b_{2}\right)
\end{aligned}
$$

4) CC5 Control Output: CC5 controls the extended carry bits $C_{5}$ and $C_{6}$ according to table 1 . Its Boolean expression is described as:

$$
\begin{aligned}
& C C 5=\sum(191,251,255) \\
& C C 5=\left(a_{3} a_{2} a_{1} a_{0} b_{3} b_{1} b_{0}+a_{3} a_{1} a_{0} b_{3} b_{2} b_{1} b_{0}\right)
\end{aligned}
$$

5) Z_ALL Control Output: Z_ALL controls generating all zeros in the sum and extended carry bits according to Table 1. Its Boolean expression is described as:

$$
\begin{gathered}
Z_{-} A L L=\sum(55,110,115,230) \\
Z_{-} A L L=\left(a_{1} b_{1}\right)\left(\overline{a_{3}} a_{0} \overline{b_{3}} b_{0}\left(a_{2} \oplus b_{2}\right)+\right. \\
\left.a_{2} \overline{a_{0}} b_{2} \overline{b_{0}}\left(a_{3} \oplus b_{3}\right)\right)
\end{gathered}
$$

The Output Generation Unit receives the control signals, (CSO, CS2. CS3, CC5, Z_ALL), from the Zero Detection Unit, the result of addition $\left(S_{0}-S_{3}\right)$ and the extended-carries $\left(C_{4}-C_{11}\right)$. Then it determines the actual Sum bits $\left(\mathrm{Sum}_{0}-\mathrm{Sum}_{3}\right)$ and the actual Extended-Carry bits $\left(T_{4}-T_{11}\right)$ according to the control signals described above.

\section{B. Decoder-Based}

The design of a nibble-size decoder-based adder involves the following steps[7]: (i) Generation of a truth table with two 4-bit operands --- operand A with $\mathrm{a}_{3} \mathrm{a}_{2} \mathrm{a}_{1} \mathrm{a}_{0}$ bits and Operand B with $b_{3} b_{2} b_{1} b_{0}$ bits --- addition of these two operands produces twelve outputs which are labeled as $\mathrm{c}_{11} \mathrm{c}_{10} \mathrm{c}_{9} \mathrm{c}_{8} \mathrm{c}_{7} \mathrm{c}_{6} \mathrm{c}_{5} \mathrm{c}_{4} \mathrm{~S}_{3} \mathrm{~s}_{2} \mathrm{~S}_{1} \mathrm{~s}_{0}$. 
The truth table (Table 2) has a total of $2^{8}=256$ minterms. (ii) We have used a $8 \times 256$ decoder to implement this truth table. For this purpose, we expressed each output in sum-of-minterms form as shown on the next page. (iii) Finally, these expressions have been implemented using the decoder and OR gates as shown in Fig. 2.

TABLE. I. APPLICATION OF THE ZERO RULE TO 4-BIT ADDITION OPERANDS

\begin{tabular}{|c|c|c|c|}
\hline $\begin{array}{l}\text { MINTERM } \\
\text { (Dec.) }\end{array}$ & $\begin{array}{l}\mathbf{a}_{3} \mathbf{a}_{2} \mathbf{a}_{1} \mathbf{a}_{0} \mathbf{b}_{3} \mathbf{b}_{2} \mathbf{b}_{1} \mathbf{b}_{0} \\
(\mathbf{H e x} .)\end{array}$ & $\begin{array}{l}\mathrm{C}_{11} \mathrm{C}_{10} \mathrm{C}_{9} \mathrm{C}_{8} \mathrm{C}_{7} \mathrm{C}_{6} \mathrm{C}_{5} \mathrm{C}_{4} \\
\text { (Hex. })\end{array}$ & $\begin{array}{l}\mathbf{S}_{3} \mathbf{S}_{2} \mathbf{S}_{1} \mathbf{S}_{0} \\
(\text { Hex. })\end{array}$ \\
\hline 55 & 37 & 00 & 0 \\
\hline 63 & $3 F$ & 00 & 8 \\
\hline 110 & $6 \mathrm{E}$ & 00 & 0 \\
\hline 111 & $6 \mathrm{~F}$ & 00 & 1 \\
\hline 115 & 73 & 00 & 0 \\
\hline 119 & 77 & 00 & 4 \\
\hline 123 & 7B & 00 & 4 \\
\hline 126 & $7 \mathrm{E}$ & 00 & 1 \\
\hline 127 & $7 \mathrm{~F}$ & 00 & C \\
\hline 183 & B7 & 00 & 8 \\
\hline 191 & $\mathrm{BF}$ & 06 & 0 \\
\hline 230 & E6 & 00 & 0 \\
\hline 231 & E7 & 00 & 1 \\
\hline 238 & EE & 00 & 8 \\
\hline 239 & $\mathrm{EF}$ & 00 & 9 \\
\hline 243 & F3 & 00 & 8 \\
\hline 246 & F6 & 00 & 1 \\
\hline 247 & F7 & 00 & C \\
\hline 251 & FB & 06 & 0 \\
\hline 254 & $\mathrm{FE}$ & 00 & 9 \\
\hline 255 & FF & 06 & 4 \\
\hline
\end{tabular}

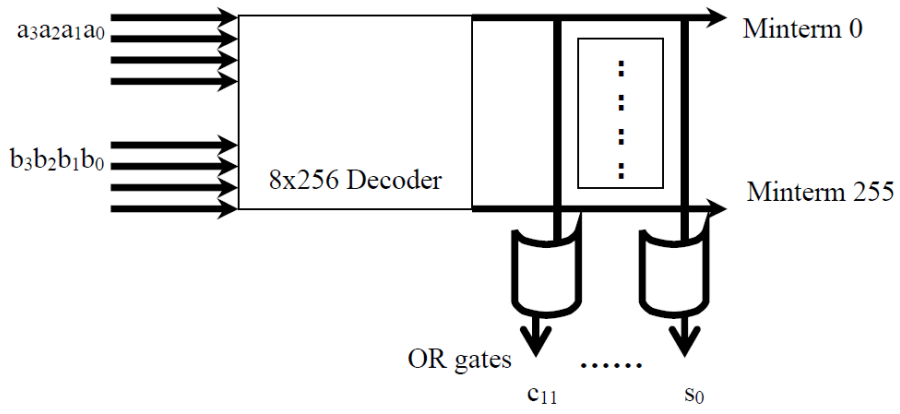

Fig. 2. Block Diagram of a 4-bit Complex Binary Complex Binary Adder using Decoder.
$\mathrm{c}_{11}=\Sigma(187) \quad \mathrm{c}_{10}=\Sigma(187) \quad \mathrm{c}_{9}=\Sigma(187)$

$\mathrm{c}_{8}=\Sigma$

$(29,31,61,89,91,93,95,121,125,149,151,157,159,181,189$, $204,205,206,207,209,211,213,215,217,219,220,221,222,223$, $236,237,241,245,249,252,253)$

$\mathrm{c}_{7}=\sum(29,31,61,89,91,93,95,102,103,118,121,125,149,151,157$, 159, 181,187,189,204, 205,206,207,209,211,213,215,217,219, $220,221,222,223,236,237,241,245,249,252,253)$

$\mathrm{c}_{6}=\Sigma(25,27,29,31,42,43,46,47,51,57,58,59,61,62,89,91,93$, $95,102,103,106,107,118,121,122,125,136,137,138,139,140$, $141,142,143,145,147,149,151,152,153,154,155,156,157,158$, $159,162,163,166,167,168,169,170,171,172,173,174,175,177$, $178,179,181,182,184,185,186,188,189,190,191,200,201,202$, $203,204,205,206,207,209,211,213,215,216,217,218,219,220$, $221,222,223,226,227,232,233,234,235,236,237,241,242,245$, $248,249,250,251,252,253,255)$

$c_{5}=\Sigma(21,23,25,27,42,43,46,47,51,53,57,58,59,62,68,69,70$, $71,76,77,78,79,81,83,84,85,86,87,92,94,100,101,102,103$, $106,107,108,109,113,116,117,118,122,124,136,137,138,139$, $140,141,142,143,145,147,152,153,154,155,156,158,162$, $163,166,167,168,169,170,171,172,173,174,175,177,178,179$, $182,184,185,186,188,190,191,196,197,198,199,200,201,202$, $203,212,214,216,218,226,227,228,229,232,233,234,235$, $242,244,248,250,251,255)$

$\mathrm{c}_{4}=\Sigma(21,23,29,31,34,35,38,39,42,43,46,47,50,51,53,54,58$, $59,61,62,68,69,70,71,76,77,78,79,81,83,84,85,86,87,89,91,92$, $93,94,95,98,99,100,101,106,107,108,109,113,114,116,117$, $121,122,124,125,149,151,157,159,162,163,166,167,170,171$, $174,175,178,179,181,182,186,187,189,190,196,197,198$, $199,204,205,206,207,209,211,212,213,214,215,217,219,220$, $221,222,223,226,227,228,229,234,235,236,237,241,242$, $244,245,249,250,252,253)$

$\mathrm{s}_{3}=\Sigma(8,9,10,11,12,13,14,15,17,19,21,23,24,26,28,30,34,35$, $38,39,40,41,44,45,49,50,53,54,56,59,60,63,72,73,74,75,76$, $77,78,79,81,83,85,87,88,90,92,94,98,99,102,103,104,105$, $108,109,113,114,117,118,120,123,124,127,128,129,130,131$, $132,133,134,135,144,146,148,150,153,155,157,159,160,161$, $164,165,170,171,174,175,176,179,180,183,185,186,189,190$, 192, 193,194,195,196,197,198,199,208,210,212,214,217,219, $221,223,224,225,228,229,234,235,238,239,240,243,244,247$, $249,250,253,254)$

$\mathrm{s}_{2}=\Sigma(4,5,6,7,12,13,14,15,17,19,20,22,25,27,28,30,36,37,38$, $39,44,45,46,47,49,51,52,54,57,59,60,62,64,65,66,67,72,73$, $74,75,80,82,85,87,88,90,93,95,96,97,98,99,104,105,106,107$, $112,114,117,119,120,122,125,127,132,133,134,135,140,141$, $142,143,145,147,148,150,153,155,156,158,164,165,166,167$, $172,173,174,175,177,179,180,182,185,187,188,190,192,193$, $194,195,200,201,202,203,208,210,213,215,216,218,221,223$, $224,225,226,227,232,233,234,235,240,242,245,247,248,250$, 253,255)

$\mathrm{s}_{1}=\Sigma(2,3,6,7,10,11,14,15,18,19,22,23,26,27,30,31,32,33,36$, $37,40,41,44,45,48,49,52,53,56,57,60,61,66,67,70,71,74,75$, $78,79,82,83,86,87,90,91,94,95,96,97,100,101,104,105,108$, $109,112,113,116,117,120,121,124,125,130,131,134,135,138$, $139,142,143,146,147,150,151,154,155,158,159,160,161,164$, 
$165,168,169,172,173,176,177,180,181,184,185,188,189,194$, 195,198,199,202, 203,206,207,210,211,214,215,218,219, $222,223,224,225,228,229,232,233,236,237,240,241,244,245$, $248,249,252,253)$

$\mathrm{s}_{0}=\Sigma(1,3,5,7,9,11,13,15,16,18,20,22,24,26,28,30,33,35,37,39$, $41,43,45,47,48,50,52,54,56,58,60,62,65,67,69,71,73,75,77,79$, $80,82,84,86,88,90,92,94,97,99,101,103,105,107,109,111,112$, $114,116,118,120,122,124,126,129,131,133,135,137,139,141$, $143,144,146,148,150,152,154,156,158,161,163,165,167,169$, $171,173,175,176,178,180,182,184,186,188,190,193,195,197$, $199,201,203,205,207,208,210,212,214,216,218,220,222,225$, $227,229,231,233,235,237,239,240,242,244,246,248,250,252$, 254)

\section{Minimum-Delay}

The truth table of the 4-bit complex binary adder, given in Table 2, was entered, one output at a time, into online Karnaugh Map [8] and simplified Boolean expression for each output was obtained. To facilitate use of online K-Map, the inputs were labeled as ABCD for the augend and EFGH for the addend. The outputs were labeled as JKLMPRSTUWYZ. The simplified expression for each output was implemented on Xilinx FPGAs and statistics for the circuit were obtained.

The simplified expressions obtained for outputs are:

$J=K=L=A \bar{B} C D E \bar{F} G H$

$$
\begin{aligned}
M=\bar{C} D E F H+ & D E F \bar{G} H+B \bar{C} D E H+A \bar{C} D F H+A D F \bar{G} H \\
& +A B \bar{C} E F+A B E F \bar{G}+A B \bar{C} D H+A B D \bar{G} H \\
P=\bar{C} D E F H+ & D E F \bar{G} H+B \bar{C} D E H+B D E \bar{G} H+A \bar{C} D F H \\
& +A D F \bar{G} H+A B \bar{C} E F+A B E F \bar{G}+A B \bar{C} D H \\
& +A B D \bar{G} H+\bar{A} B C \overline{D E} F G+\bar{A} B C \bar{E} F G \bar{H} \\
& +A \bar{B} C D E \bar{F} G H
\end{aligned}
$$

$$
\begin{aligned}
R=A \bar{C} E+A E \bar{G} & +\bar{C} D E H+D E \bar{G} H+A \bar{C} D H+A D \bar{G} H \\
& +A B E \bar{F}+\bar{B} C \bar{D} E G+C \bar{D} E \bar{F} G+\bar{B} C E G \bar{H} \\
& +C E \bar{F} G \bar{H}+A D E F H+A \bar{B} C \bar{D} G+A C \overline{D F} G \\
& +A \bar{B} C G \bar{H}+A C \bar{F} G \bar{H}+A \bar{B} D \overline{E F} H \\
& +\overline{A B} C D \bar{F} G H+\bar{A} B C \overline{D E} F G+\bar{A} B C \bar{E} F G \bar{H}
\end{aligned}
$$

$$
\begin{aligned}
S=A \overline{B D} E+A \bar{D} & \bar{F}+A \bar{B} E \bar{H}+A E \overline{F H}+\bar{B} C \bar{D} E G \\
& +C \bar{D} E \bar{F} G+\bar{B} C E G \bar{H}+C E \bar{F} G \bar{H}+\bar{A} B \overline{C D} F \\
& +\bar{A} B \overline{D E} F+B \bar{C} \overline{D \bar{E}} F+\bar{A} B \bar{D} F \bar{G} \\
& +B \overline{D E} F \bar{G}+\bar{A} B \bar{C} \bar{F}+\bar{A} B \bar{E} F \bar{H} \\
& +B \overline{C E} F \bar{H}+\bar{A} B F \overline{G H}+B \bar{E} F \overline{G H} \\
& +A \overline{B C} E \bar{F}+A \bar{B} E \overline{F G}+A \bar{B} C \bar{D} G+A C \overline{D F} G \\
& +A \bar{B} C G \bar{H}+A C \bar{F} G \bar{H}+\overline{A C} D \bar{E} F H \\
& +\bar{A} D \bar{E} F \bar{G} H+\overline{A B} D E \bar{F} H+A \bar{B} D \overline{E F} H \\
& +A B C E \bar{F} G+\overline{A B} C D \bar{F} G H+\bar{A} B \overline{C D E G} H \\
& +\bar{A} B \bar{C} D \bar{E} G H+\bar{A} B C D \overline{E G} H+A C D E F G H
\end{aligned}
$$

$$
\begin{aligned}
T=B \bar{C} F+B F \bar{G} & +\bar{C} D F H+D F \bar{G} H+\bar{B} C \bar{D} G+\bar{B} C \bar{F} G \\
& +C \overline{D F} G+\bar{B} C G \bar{H}+C \bar{F} G \bar{H}+B \bar{C} D G H \\
& +B \bar{C} D E H+B C D \bar{G} H+A B \bar{C} D H \\
& +\bar{A} B \overline{C D E G} H \\
U=\overline{A C D} E+\overline{A D} & \bar{G}+\overline{A C} E \bar{H}+\bar{A} E \overline{G H}+A \bar{C} D E+A \overline{D E G} \\
& +A \overline{C E H}+A \overline{E G H}+\overline{A C} D \bar{E} H+\bar{A} D \overline{E G} H \\
& +\bar{A} C \overline{D E} G+\bar{A} C \bar{E} G \bar{H}+A \bar{C} D E H \\
& +A D E \bar{G} H+A C \bar{D} E G+A C E G \bar{H} \\
& +\bar{A} C D E G H+A C D \bar{E} G H+\bar{A} B \overline{C D F G} H \\
W & \\
W \overline{B D} F+B \overline{F H} & +\overline{A B} \bar{F} \bar{H}+\overline{B E} F \bar{H}+\bar{B} F G \bar{H}+\bar{B} D \bar{F} H \\
& +\bar{B} C F \bar{H}+B \overline{D F} G+B \bar{D} E \bar{F}+B D F H \\
& +B C \overline{D F}+A B \overline{D F}+\overline{A C} D \overline{E F G} H \\
& +A \overline{B C} E \bar{G} H \\
Y=\bar{C} G+C \bar{G}+ & \bar{A} B \overline{C E F} H \\
Z=D \bar{H}+\overline{B D} H & +\bar{D} G H+\bar{D} F H+\bar{D} E H+C \bar{D} H+A \bar{D} H \\
& +\bar{A} B \bar{C} D \overline{E F G}
\end{aligned}
$$

The logic diagram of minimum-delay complex binary adder is given in Fig. 3.

The logic diagram of each adder output (J,K,L,M,P,R,S,T, $\mathrm{U}, \mathrm{W}, \mathrm{Y}, \mathrm{Z})$ is shown in Figs.4-15.

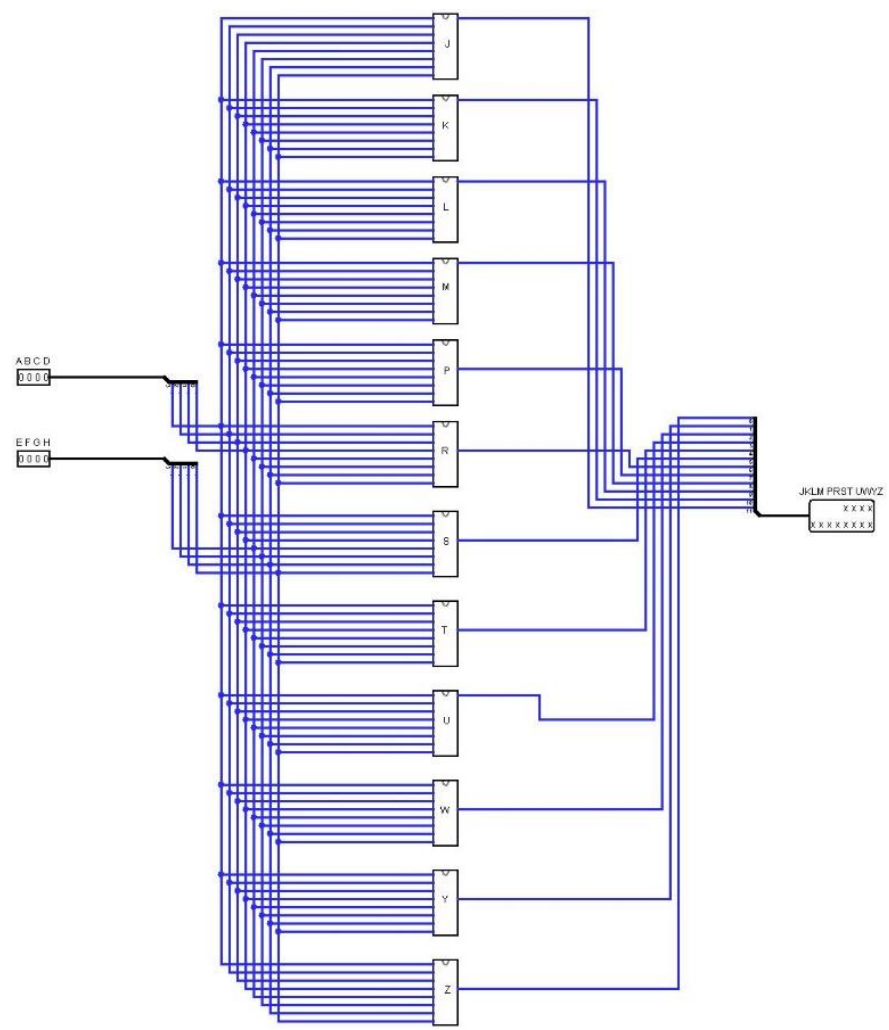

Fig. 3. Block Diagram of a 4-bit Complex Binary Minimum-Delay Adder. 
TABLE. II. TRUTH TABLE OF A 4-BIT COMPLEX BINARY ADDER (MINTERM: $\mathrm{A}_{3} \mathrm{~A}_{2} \mathrm{~A}_{1} \mathrm{~A}_{0}$ ADD B ${ }_{3} \mathrm{~B}_{2} \mathrm{~B}_{1} \mathrm{~B}_{0}=\mathrm{C}_{11} \mathrm{C}_{10} \mathrm{C}_{9} \mathrm{C}_{8} \mathrm{C}_{7} \mathrm{C}_{6} \mathrm{C}_{5} \mathrm{C}_{4} \mathrm{~S}_{3} \mathrm{~S}_{2} \mathrm{~S}_{1} \mathrm{~S}_{0}$ )

\begin{tabular}{|c|c|c|c|}
\hline 0 & 00000000000000000000 & 64 & 01000000000000000100 \\
\hline 1 & 00000001000000000001 & 65 & 01000001000000000101 \\
\hline 2 & 00000010000000000010 & 66 & 01000010000000000110 \\
\hline 3 & 00000011000000000011 & 67 & 01000011000000000111 \\
\hline 4 & 00000100000000000100 & 68 & 01000100000000110000 \\
\hline 5 & 00000101000000000101 & 69 & 01000101000000110001 \\
\hline 6 & 00000110000000000110 & 70 & 01000110000000110010 \\
\hline 7 & 00000111000000000111 & 71 & 01000111000000110011 \\
\hline 8 & 00001000000000001000 & 72 & 01001000000000001100 \\
\hline 9 & 00001001000000001001 & 73 & 01001001000000001101 \\
\hline 10 & 00001010000000001010 & 74 & 01001010000000001110 \\
\hline 11 & 00001011000000001011 & 75 & 01001011000000001111 \\
\hline 12 & 00001100000000001100 & 76 & 01001100000000111000 \\
\hline 13 & 00001101000000001101 & 77 & 01001101000000111001 \\
\hline 14 & 00001110000000001110 & 78 & 01001110000000111010 \\
\hline 15 & 00001111000000001111 & 79 & 01001111000000111011 \\
\hline 16 & 00010000000000000001 & 80 & 01010000000000000101 \\
\hline 17 & 00010001000000001100 & 81 & 01010001000000111000 \\
\hline 18 & 00010010000000000011 & 82 & 01010010000000000111 \\
\hline 19 & 00010011000000001110 & 83 & 01010011000000111010 \\
\hline 20 & 00010100000000000101 & 84 & 01010100000000110001 \\
\hline 21 & 00010101000000111000 & 85 & 01010101000000111100 \\
\hline 22 & 00010110000000000111 & 86 & 01010110000000110011 \\
\hline 23 & 00010111000000111010 & 87 & 01010111000000111110 \\
\hline 24 & 00011000000000001001 & 88 & 01011000000000001101 \\
\hline 25 & 00011001000001100100 & 89 & 01011001000111010000 \\
\hline 26 & 00011010000000001011 & 90 & 01011010000000001111 \\
\hline 27 & 00011011000001100110 & 91 & 01011011000111010010 \\
\hline 28 & 00011100000000001101 & 92 & 01011100000000111001 \\
\hline 29 & 00011101000111010000 & 93 & 01011101000111010100 \\
\hline 30 & 00011110000000001111 & 94 & 01011110000000111011 \\
\hline 31 & 00011111000111010010 & 95 & 01011111000111010110 \\
\hline 32 & 00100000000000000010 & 96 & 01100000000000000110 \\
\hline 33 & 00100001000000000011 & 97 & 01100001000000000111 \\
\hline 34 & 00100010000000011000 & 98 & 01100010000000011100 \\
\hline 35 & 00100011000000011001 & 99 & 01100011000000011101 \\
\hline 36 & 00100100000000000110 & 100 & 01100100000000110010 \\
\hline 37 & 00100101000000000111 & 101 & 01100101000000110011 \\
\hline 38 & 00100110000000011100 & 102 & 01100110000011101000 \\
\hline 39 & 00100111000000011101 & 103 & 01100111000011101001 \\
\hline 40 & 00101000000000001010 & 104 & 01101000000000001110 \\
\hline 41 & 00101001000000001011 & 105 & 01101001000000001111 \\
\hline 42 & 00101010000001110000 & 106 & 01101010000001110100 \\
\hline 43 & 00101011000001110001 & 107 & 01101011000001110101 \\
\hline 44 & 00101100000000001110 & 108 & 01101100000000111010 \\
\hline 45 & 00101101000000001111 & 109 & 01101101000000111011 \\
\hline 46 & 00101110000001110100 & 110 & 01101110000000000000 \\
\hline 47 & 00101111000001110101 & 111 & 01101111000000000001 \\
\hline 48 & 00110000000000000011 & 112 & 01110000000000000111 \\
\hline 49 & 00110001000000001110 & 113 & 01110001000000111010 \\
\hline 50 & 00110010000000011001 & 114 & 01110010000000011101 \\
\hline 51 & 00110011000001110100 & 115 & 01110011000000000000 \\
\hline 52 & 00110100000000000111 & 116 & 01110100000000110011 \\
\hline 53 & 00110101000000111010 & 117 & 01110101000000111110 \\
\hline 54 & 00110110000000011101 & 118 & 01110110000011101001 \\
\hline 55 & 00110111000000000000 & 119 & 01110111000000000100 \\
\hline 56 & 00111000000000001011 & 120 & 01111000000000001111 \\
\hline 57 & 00111001000001100110 & 121 & 01111001000111010010 \\
\hline 58 & 00111010000001110001 & 122 & 01111010000001110101 \\
\hline 59 & 00111011000001111100 & 123 & 01111011000000001000 \\
\hline 60 & 00111100000000001111 & 124 & 01111100000000111011 \\
\hline 61 & 00111101000111010010 & 125 & 01111101000111010110 \\
\hline 62 & 00111110000001110101 & 126 & 01111110000000000001 \\
\hline 63 & 00111111000000001000 & 127 & 01111111000000001100 \\
\hline
\end{tabular}

\begin{tabular}{|c|c|c|c|}
\hline 128 & 10000000000000001000 & 192 & 11000000000000001100 \\
\hline 129 & 1000000100000001001 & 193 & 11000001000000001101 \\
\hline 130 & 1000001000000001010 & 194 & 11000010000000001110 \\
\hline 131 & 1000001100000001011 & 195 & 11000011000000001111 \\
\hline 132 & 1000010000000001100 & 196 & 11000100000000111000 \\
\hline 133 & 1000010100000001101 & 197 & 11000101000000111001 \\
\hline 134 & 1000011000000001110 & 198 & 11000110000000111010 \\
\hline 135 & 10000111000000001111 & 199 & 11000111000000111011 \\
\hline 136 & 10001000000001100000 & 200 & 11001000000001100100 \\
\hline 137 & 10001001000001100001 & 201 & 11001001000001100101 \\
\hline 138 & 10001010000001100010 & 202 & 11001010000001100110 \\
\hline 139 & 10001011000001100011 & 203 & 11001011000001100111 \\
\hline 140 & 10001100000001100100 & 204 & 11001100000111010000 \\
\hline 141 & 10001101000001100101 & 205 & 11001101000111010001 \\
\hline 142 & 10001110000001100110 & 206 & 11001110000111010010 \\
\hline 143 & 10001111000001100111 & 207 & 11001111000111010011 \\
\hline 144 & 10010000000000001001 & 208 & 11010000000000001101 \\
\hline 145 & 10010001000001100100 & 209 & 11010001000111010000 \\
\hline 146 & 1001001000000001011 & 210 & 11010010000000001111 \\
\hline 147 & 10010011000001100110 & 211 & 11010011000111010010 \\
\hline 148 & 1001010000000001101 & 212 & 11010100000000111001 \\
\hline 149 & 10010101000111010000 & 213 & 11010101000111010100 \\
\hline 150 & 1001011000000001111 & 214 & 11010110000000111011 \\
\hline 151 & 10010111000111010010 & 215 & 11010111000111010110 \\
\hline 152 & 10011000000001100001 & 216 & 11011000000001100101 \\
\hline 153 & 10011001000001101100 & 217 & 11011001000111011000 \\
\hline 154 & 10011010000001100011 & 218 & 11011010000001100111 \\
\hline 155 & 10011011000001101110 & 219 & 11011011000111011010 \\
\hline 156 & 10011100000001100101 & 220 & 11011100000111010001 \\
\hline 157 & 10011101000111011000 & 221 & 11011101000111011100 \\
\hline 158 & 10011110000001100111 & 222 & 11011110000111010011 \\
\hline 159 & 10011111000111011010 & 223 & 11011111000111011110 \\
\hline 160 & 10100000000000001010 & 224 & 11100000000000001110 \\
\hline 161 & 10100001000000001011 & 225 & 11100001000000001111 \\
\hline 162 & 10100010000001110000 & 226 & 11100010000001110100 \\
\hline 163 & 10100011000001110001 & 227 & 11100011000001110101 \\
\hline 164 & 1010010000000001110 & 228 & 11100100000000111010 \\
\hline 165 & 1010010100000001111 & 229 & 11100101000000111011 \\
\hline 166 & 10100110000001110100 & 230 & 11100110000000000000 \\
\hline 167 & 10100111000001110101 & 231 & 11100111000000000001 \\
\hline 168 & 10101000000001100010 & 232 & 11101000000001100110 \\
\hline 169 & 10101001000001100011 & 233 & 11101001000001100111 \\
\hline 170 & 10101010000001111000 & 234 & 11101010000001111100 \\
\hline 171 & 10101011000001111001 & 235 & 11101011000001111101 \\
\hline 172 & 10101100000001100110 & 236 & 11101100000111010010 \\
\hline 173 & 10101101000001100111 & 237 & 11101101000111010011 \\
\hline 174 & 10101110000001111100 & 238 & 11101110000000001000 \\
\hline 175 & 10101111000001111101 & 239 & 11101111000000001001 \\
\hline 176 & 1011000000000001011 & 240 & 11110000000000001111 \\
\hline 177 & 10110001000001100110 & 241 & 11110001000111010010 \\
\hline 178 & 10110010000001110001 & 242 & 11110010000001110101 \\
\hline 179 & 10110011000001111100 & 243 & 11110011000000001000 \\
\hline 180 & 1011010000000001111 & 244 & 11110100000000111011 \\
\hline 181 & 10110101000111010010 & 245 & 11110101000111010110 \\
\hline 182 & 10110110000001110101 & 246 & 11110110000000000001 \\
\hline 183 & 10110111000000001000 & 247 & 11110111000000001100 \\
\hline 184 & 10111000000001100011 & 248 & 11111000000001100111 \\
\hline 185 & 10111001000001101110 & 249 & 11111001000111011010 \\
\hline 186 & 10111010000001111001 & 250 & 11111010000001111101 \\
\hline 187 & 10111011111010010100 & 251 & 11111011000001100000 \\
\hline 188 & 10111100000001100111 & 252 & 11111100000111010011 \\
\hline 189 & 10111101000111011010 & 253 & 11111101000111011110 \\
\hline 190 & 10111110000001111101 & 254 & 11111110000000001001 \\
\hline 191 & 10111111000001100000 & 255 & 11111111000001100100 \\
\hline
\end{tabular}




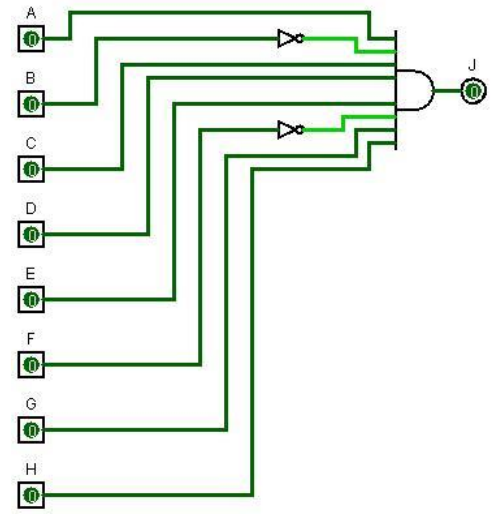

Fig. 4. Logic Diagram for Output J.

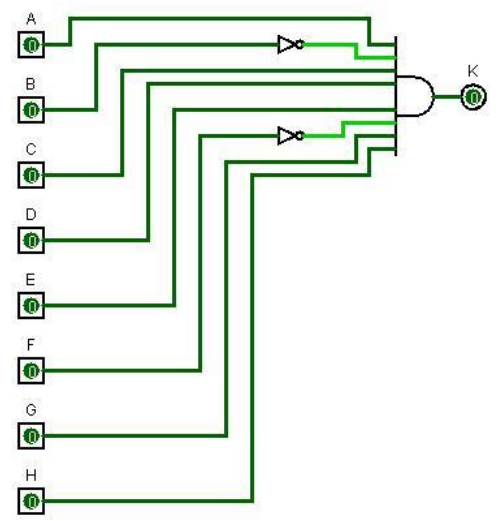

Fig. 5. Logic Diagram for Output K.

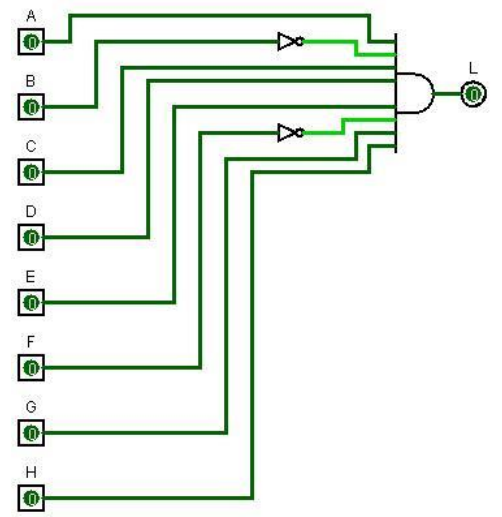

Fig. 6. Logic Diagram for Output L.

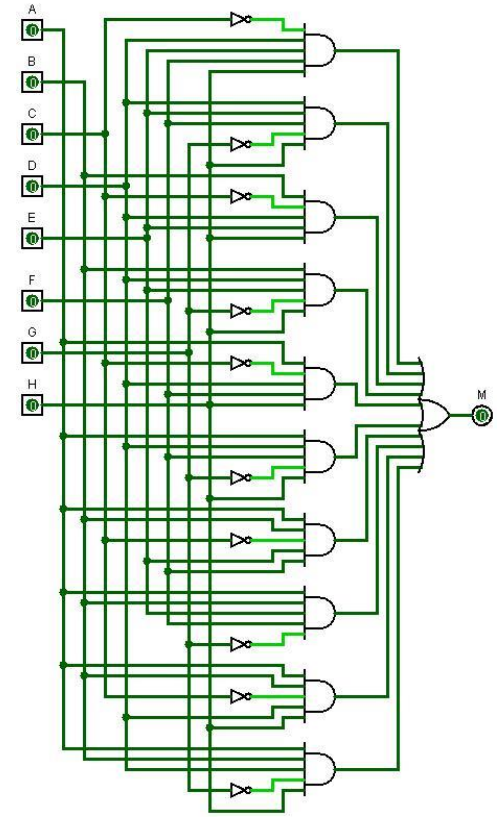

Fig. 7. Logic Diagram for Output M.

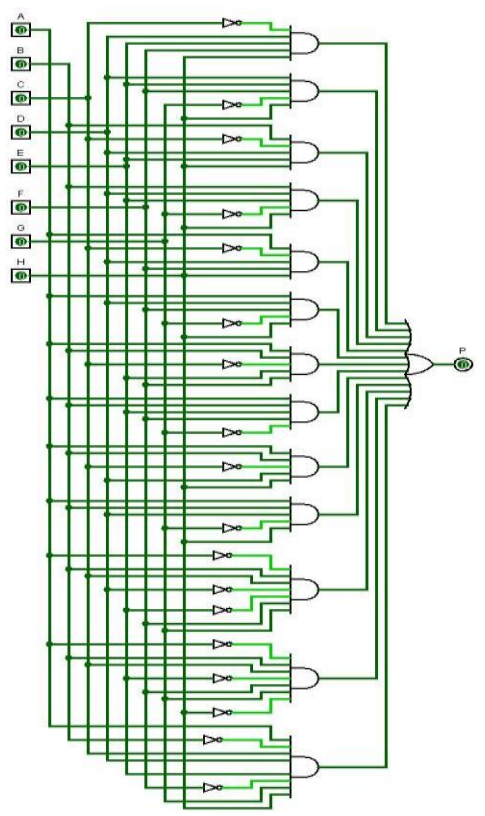

Fig. 8. Logic Diagram for Output P.

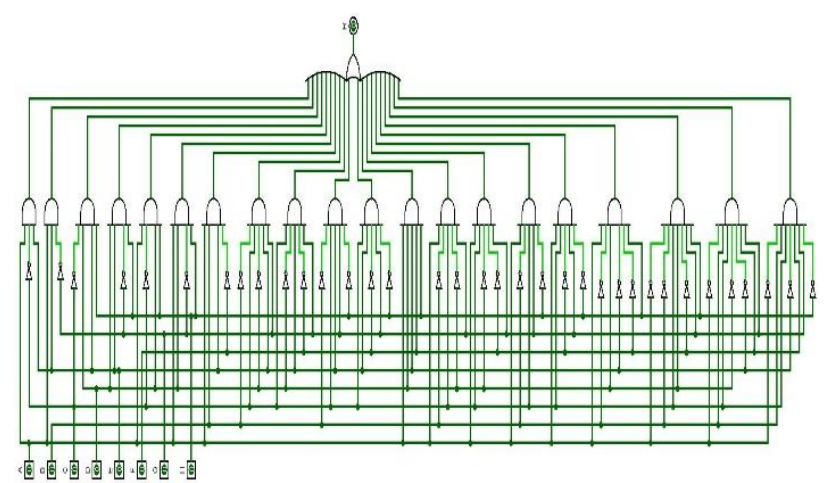

Fig. 9. Logic Diagram for Output R. 


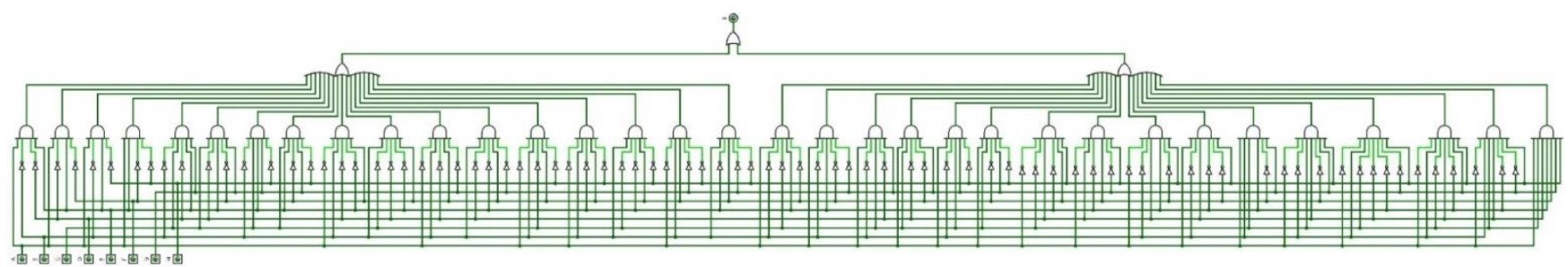

Fig. 10. Logic diagram for Output $\mathrm{S}$

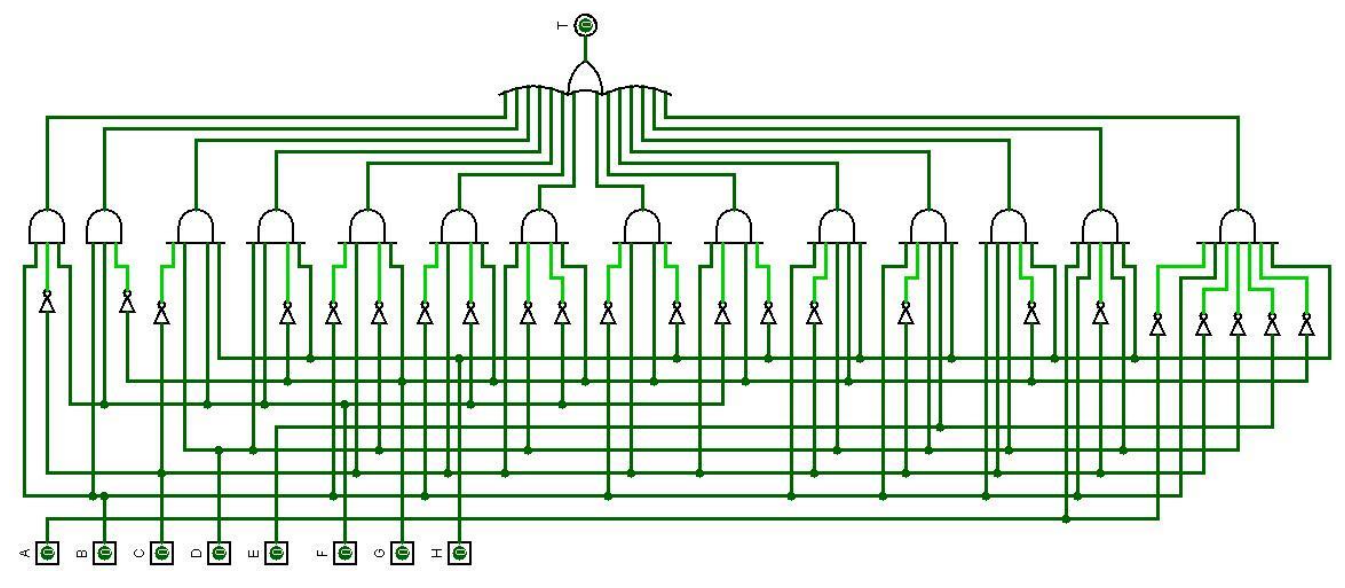

Fig. 11. Logic diagram for Output $\mathrm{T}$

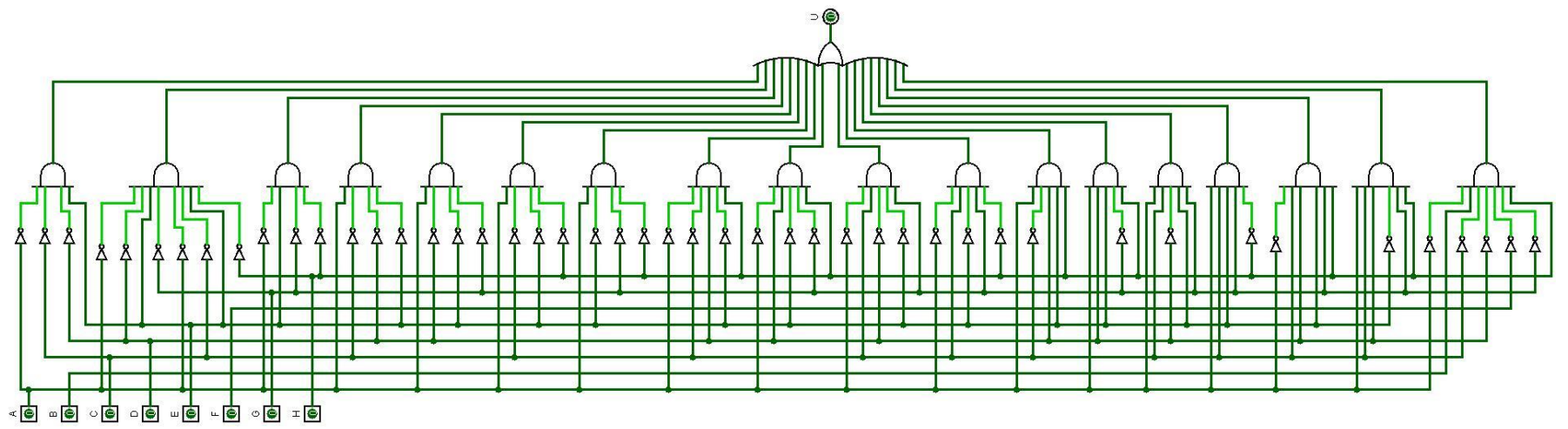

Fig. 12. Logic diagram for Output $U$

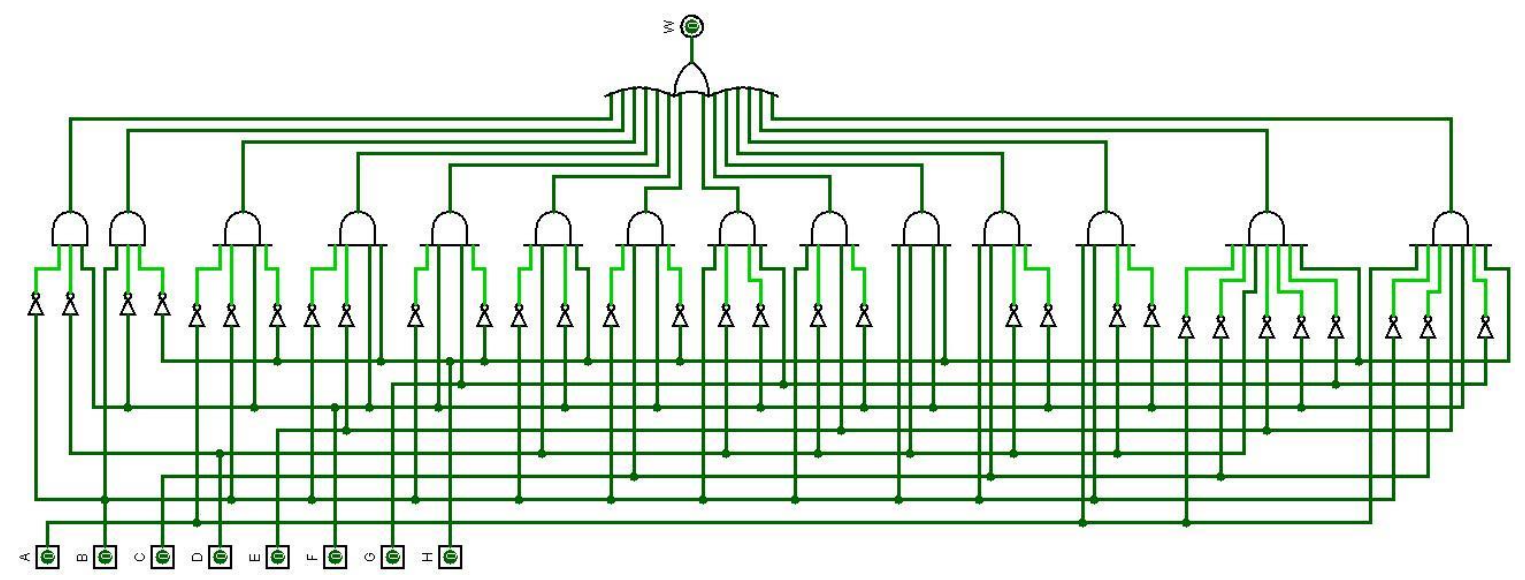

Fig. 13. Logic Diagram for Output W. 


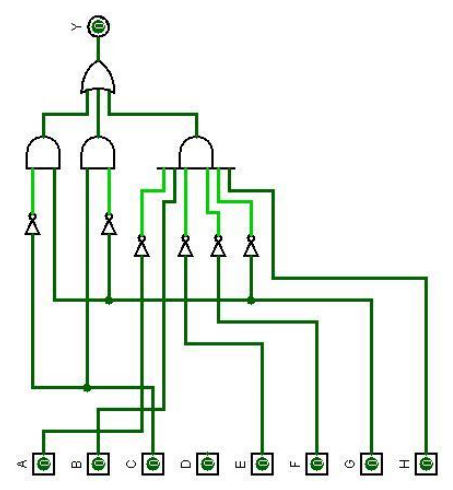

Fig. 14. Logic diagram for Output Y.

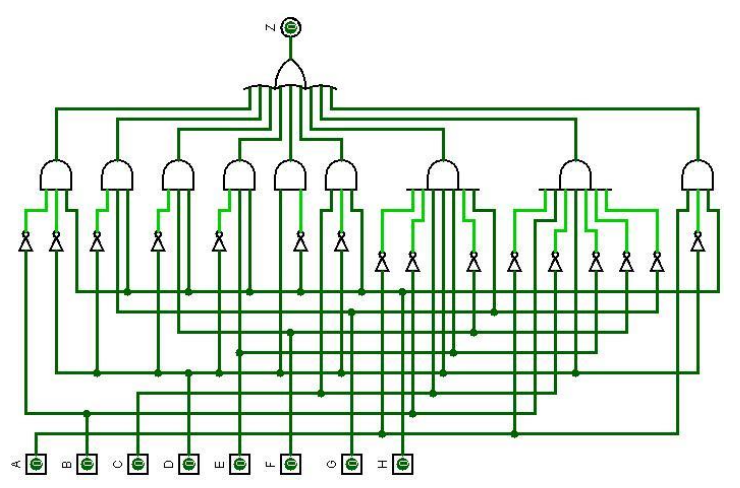

Fig. 15. Logic Diagram for Output Z.

\section{RESULTS}

Complex binary adder designs presented in the previous section have been implemented on Xilinx[9] FPGAs and various statistics pertaining to each design are given in Tables 3-5.

TABLE. III. FPGA IMPLEMENTATION RESUlts OF COMPLEX BinARy RIPPLE-CARRy ADDER (CBRCA) AND BASE 2 AdDER [5]

\begin{tabular}{|c|c|c|c|c|}
\hline & \multicolumn{3}{|c|}{ Complex Binary Ripple-Carry Adder Implementations on FPGA Devices } & \multirow{2}{*}{$\begin{array}{l}\text { Base } 2 \text { Ripple-Carry Adder } \\
\text { Implementation }\end{array}$} \\
\hline & XC 4003E & Spartan XCS05 & Virtex XCV50 & \\
\hline Number of external IOBs ${ }^{a}$ & $20 / 80(32 \%)$ & $20 / 80(32 \%)$ & $20 / 94(21 \%)$ & $13 / 94(13 \%)$ \\
\hline Number of CLBs ${ }^{\mathrm{b}}\left(\right.$ Slices $\left.^{\mathrm{c}}\right)$ & $24 / 100(24 \%)$ & $24 / 100(24 \%)$ & $31 / 768(4 \%)$ & $6 / 768(1 \%)$ \\
\hline Number of 4 input LUTs ${ }^{\mathrm{d}}$ & $42 / 200(21 \%)$ & $42 / 200(21 \%)$ & $59 / 1536(3 \%)$ & $9 / 1536(1 \%)$ \\
\hline Number of 3 input LUTs & $13 / 100(13 \%)$ & $13 / 100(13 \%)$ & & \\
\hline Number of bonded IOBs & $20 / 61(32 \%)$ & $20 / 61(32 \%)$ & $20 / 94(21 \%)$ & $13 / 94(13 \%)$ \\
\hline Gate count & 310 & 310 & 354 & 54 \\
\hline Average connection delay (ns) & 2.808 & 3.506 & 1.640 & 1.525 \\
\hline Maximum combinational delay (ns) & 35.680 & 45.995 & 24.839 & 15.389 \\
\hline
\end{tabular}

TABLE. IV. FPGA IMPLEMENTATIOn ReSults of COMPLEX BinARy DECODER-BASEd AdDER AND baSe 2 AdDER [5]

\begin{tabular}{|l|l|l|}
\hline & \multicolumn{1}{|c|}{$\begin{array}{c}\text { Complex Binary Decoder-based Adder Implementation on } \\
\text { FPGA Device }\end{array}$} & Base 2 Decoder-based Adder Implementation \\
\hline & Virtex V50CS144 & Virtex V50CS144 \\
\hline Number of external IOBs & $20 / 94(21 \%)$ & $33 / 94(13 \%)$ \\
\hline Number of CLBs(Slices) & $455 / 768(59 \%)$ & $755 / 1536(49 \%)$ \\
\hline Number of 4 input LUTs & $857 / 1536(55 \%)$ & $13 / 94(13 \%)$ \\
\hline Number of bonded IOBs & $20 / 94(21 \%)$ & 4530 \\
\hline Gate count & 5142 & 3.169 \\
\hline Average connection delay (ns) & 3.179 & 28.442 \\
\hline Maximum combinational delay (ns) & 32.471 & \\
\hline
\end{tabular}


TABLE. V. FPGA IMPLEMENTATION RESULTS OF COMPLEX BINARY MINIMUM-DELAY ADDER [10]

\begin{tabular}{|l|l|l|l|}
\hline & \multicolumn{3}{|c|}{$\begin{array}{c}\text { Complex Binary Minimum-Delay Adder } \\
\text { Implementations on FPGA Devices }\end{array}$} \\
\hline & $\begin{array}{l}\text { Virtex4 } \\
\text { XC4VLX15 }\end{array}$ & $\begin{array}{l}\text { Virtex5 } \\
\text { XC5VLX30 }\end{array}$ & $\begin{array}{l}\text { Virtex } \\
\text { XCV100 }\end{array}$ \\
\hline $\begin{array}{l}\text { Number of } \\
\text { external IOBs }\end{array}$ & $20 / 240(8 \%)$ & $20 / 220(9 \%)$ & $\begin{array}{l}20 / 180 \\
(11 \%)\end{array}$ \\
\hline $\begin{array}{l}\text { Number of } \\
\text { CLBs(Slices) }\end{array}$ & $27 / 6144(>1 \%)$ & $25 / 19200(1 \%)$ & $\begin{array}{l}27 / 1200 \\
(2 \%)\end{array}$ \\
\hline $\begin{array}{l}\text { Number of 4 input } \\
\text { LUTs }\end{array}$ & $52 / 12288(>1 \%)$ & $0 / 27$ & $\begin{array}{l}52 / 2400 \\
(2 \%)\end{array}$ \\
\hline $\begin{array}{l}\text { Number of } \\
\text { bonded IOBs }\end{array}$ & $20 / 240(6 \%)$ & $20 / 220(9 \%)$ & $\begin{array}{l}20 / 180 \\
(11 \%)\end{array}$ \\
\hline Gate count & 330 & 175 & 330 \\
\hline $\begin{array}{l}\text { Maximum net } \\
\text { delay (ns) }\end{array}$ & 5.028 & 2.790 & 8.856 \\
\hline $\begin{array}{l}\text { Maximum } \\
\text { combinational } \\
\text { delay (ns) }\end{array}$ & 7.827 & 4.776 & 17.001 \\
\hline
\end{tabular}

\section{ACKNOWLEDGMENT}

We gratefully acknowledge the support provided by Sultan Qaboos University through Internal Research Grant No. IG/ENG/IENG/16/03 in facilitating and encouraging an environment conducive to research and academic excellence in the Sultanate of Oman.

\section{REFERENCES}

[1] T. Jamil, N. Holmes, and D. Blest:`Towards Implementation of a Binary Number System for Complex Numbers', Proceedings of the IEEE SoutheastCon 2000, Nashville, Tennessee (USA), April 7-9, 2000, pp. 268-274.

[2] T. Jamil, "The complex binary number system - basic arithmetic made simple," IEEE Potentials, Vol. 20, No. 5, pp. 39-41, 2002.

[3] D. Blest and T. Jamil, "Division in a binary representation for complex numbers," International Journal of Mathematical Education in Science and Technology, Vol. 34, No. 4, pp, 561-574, 2003.

[4] T. Jamil, "Complex Binary Number System - Algorithms and Circuits," Springer, 2013.

[5] T. Jamil, B. Arafeh, and A. AlHabsi, "Hardware Implementation and Performance Evaluation of Complex Binary Adder Designs," Proceedings of the 7th World Multiconference on Systemics, Cybernetics, and Informatics (SCI 2003), Orlando, Florida (USA), 27-30 July 2003, Vol. II, pp. 68-73.

[6] B. Arafeh, T. Jamil, and A. AlHabsi, "A Nibble-size Ripple-carry Adder for $(-1+\mathrm{j})$-base Complex Binary Numbers," Proceedings of the International Arab Conference on Information Technology (ACIT 2002), Doha (Qatar), 16-19 December 2002, Vol. I, pp. 207-211.

[7] T. Jamil, B. Arafeh, and A. AlHabsi, "Design of a Nibble-size Adder for $(-1+\mathrm{j})$-base Complex Binary Numbers," Proceedings of the 6th World Multiconference on Systemics, Cybernetics, and Informatics (SCI 2002), Orlando, Florida (USA), 14-18 July 2002, Vol. V, pp. 297-302.

[8] www.32x8.com

[9] www.xilinx.com

[10] T. Jamil, M. Awadalla, and I. Mohammed, "Design and Implementation of a Complex Binary Adder," Medwell Journal of Engineering and Applied Sciences, ISSN: 1818-7803, Vol. 13, No. 7, 2018, pp. 18131828. 